\title{
Integración social: ¿oportunidad de que familias de escasos recursos vivan en sectores de mayores ingresos y equipamientos? Una mirada a las posibilidades que entregan el mercado, el Estado y la vía de la informalidad
}

Andrés Godoy. тесно-Chile, Santiago, Chile.

RESUMEN | El presente artículo tiene por objetivo analizar las opciones de integración territorial existentes en la ciudad de Santiago, respecto a la posibilidad de que familias de escasos recursos obtengan una vivienda en comunas con buenas condiciones de calidad de vida urbana y que, además, no congreguen grandes masas de familias vulnerables en un mismo territorio. Tal análisis se realiza mediante la construcción de datos cuantitativos relativos a las oportunidades existentes en la actualidad para acceder a una vivienda, ya sea mediante el mercado privado, el apoyo estatal o acciones informales. Se concluye que ninguno de estos caminos permite mejorar la integración territorial de las familias más vulnerables del país. Queda, entonces, la interrogante respecto a por qué el Estado no promueve con mayor fuerza mecanismos que permitan lograr tal objetivo, tomando en consideración los diferentes beneficios que, según diferentes expertos, este proceso comporta.

PALABRAS CLAVE | segregación, integración territorial, vivienda.

ABSTRACT | The objective of this article is to analyze the options of territorial integration that exist in the city of Santiago, with respect to the possibility that low-income families can obtain housing in communes with good conditions of quality of urban life and that also do not congregate large masses of vulnerable families living in the same territory. The above, studied through the construction of quantitative data, graph the opportunities that currently exist to access a home, either through the private market, state support or informal actions. The data analysis concludes that none of these roads allows improving the territorial integration of the most vulnerable families in the country, leaving the question as to why the State does not promote with greater force mechanisms that allow achieving this objective, taking into account the different benefits that this process brings according to different experts.

KEYwORDs $\mid$ segregation, territorial integration, housing. 


\section{Introducción}

Diferentes instituciones, tales como el Consejo Nacional de Desarrollo Urbano (CNDU), a través de la Política Nacional de Desarrollo Urbano, 2014; el Programa de las Naciones Unidas para el Desarrollo (PNUD), a partir del libro Desiguales, 2017; o la Pontificia Universidad Católica de Chile (PUC), mediante la publicación anual del Índice de Calidad de Vida Urbana de Comunas y Ciudades de Chile (ICVU), han detectado que una de las más importantes problemáticas que se arrastra en las grandes ciudades del país, y en especial en la Región Metropolitana, es la segregación territorial.

Este fenómeno es entendido como "formas de desigual distribución de grupos de población en el territorio, que se manifiestan de diferentes maneras" (Rodríguez \& Arriagada, 2004, p. 5), ya sea mediante la distancia física residencial entre los diferentes grupos socioeconómicos, la homogeneidad social de los diferentes territorios bajo los que se organiza una sociedad, y la concentración de grupos en sectores específicos de una ciudad. Para efectos de este estudio, el elemento discriminador que se evalúa es el nivel socioeconómico de los diferentes actores de un territorio, dado que también sería posible hablar de segregación territorial por nacionalidad, religión u otros factores culturales.

En esta línea, los resultados de la Encuesta de Caracterización Socioeconómica (Casen) correspondientes a 2015 (Ministerio de Desarrollo Social, 2015) muestran la existencia de comunas en la ciudad de Santiago donde más del 10\% de familias vive bajo la línea de la pobreza por ingresos (El Bosque, Recoleta, La Pintana), versus comunas en la que prácticamente no existen familias que viven bajo la línea de la pobreza por ingresos (Las Condes, Providencia, Vitacura).

Diversos autores ya han comprobado y profundizado en dicha problemática y sus consecuencias (Rodríguez \& Arriagada, 2004; CNDU, 2014; Rodríguez \& Winchester, 2001; Sabatini, Cáceres \& Cerda, 2001). Por tal razón, el presente escrito se concentra en conocer las posibilidades actuales de romper con la segregación territorial, y solo se presenta un breve resumen de los principales efectos que genera la segregación territorial que se vive en el país y, en particular, en la ciudad de Santiago. Posteriormente, se desarrolla una breve reflexión, tomando en consideración a los mismos autores señalados, respecto de los beneficios que conlleva terminar con la segregación y producir procesos de integración territorial y social. Por último, se realiza una radiografía de la ciudad de Santiago en relación con las posibilidades de los grupos más segregados de la sociedad en cuanto a dejar de vivir en sus actuales territorios y trasladarse a comunas con mejores equipamientos urbanos y menor concentración de pobreza. Lo anterior se lleva a cabo a partir de datos cuantitativos relativos a sus ingresos y a los costos del mercado de viviendas, las oportunidades que les ofrecen los subsidios estatales, o las posibilidades de acceso a mejores viviendas a través de acciones ilegales, como las tomas de terreno.

Antes de iniciar el análisis, es necesario dejar de manifiesto ciertas precauciones y posiciones teóricas que se adoptaron al momento de hablar de la ciudad de Santiago. En primer lugar, al analizar la segregación territorial en función de los niveles socioeconómicos, se toman en consideración los planteamientos de Brain 
y Sabatini (2008), quienes mencionan la ingenuidad y simpleza en la que caen diferentes estudios urbanísticos al correlacionar de forma directa los conceptos de desigualdad socioeconómica y segregación territorial. Sostienen dichos autores que esta relación no está determinada de forma lineal, dado que diferentes sociedades en el mundo presentan altos niveles de desigualdad, pero no así en cuanto a segregación territorial; del mismo modo, existirían diferentes ciudades del mundo con menores niveles de desigualdad socioeconómica que las chilenas, pero sí con altos niveles de segregación. No obstante, ciudades como Santiago sí cuentan con altos grados de diferencia socioeconómica que se traducen en segregación territorial. Ello no responde necesariamente a la desigualdad en sí; por el contrario, es probable que estos efectos se den en función de la conformación de las ciudades y de la forma en que ellas se van constituyendo con el paso del tiempo, a partir de factores como la planificación urbana o el tipo de política de vivienda que se desarrolle.

Como segundo punto, se plantea que la complejidad de las ciudades y de las sociedades del siglo xxi no permite consideraciones simplistas al momento del análisis, como la visión de la ciudad dual en función de una clase alta y una clase obrera. Álvarez (2013) plantea que hay "dificultad para asegurar con propiedad si Santiago es una ciudad dual o medianizada, lo cual se observa también al momento de interpretar la heterogeneidad de la actual estructura social de la sociedad chilena y el papel que juega en ello lo que continuamos llamando la clase media” (p. 268).

\section{Principales efectos de la segregación territorial en la ciudad de Santiago}

\section{Desigualdad territorial:}

\section{falta de planificación urbana y políticas de vivienda deficientes}

Santiago de Chile, no cabe duda, es una ciudad segregada territorialmente en función de los niveles socioeconómicos de sus habitantes. No obstante, esta división de grupos en cuanto al espacio no solo ha implicado aglomeraciones de pobreza o falta de interacción entre grupos distintos. Además, ha conllevado una diferencia objetiva y material entre ellos, la cual se ve reflejada en la desigualdad territorial de equipamientos y servicios y en la calidad de estos, visible al compararse las distintas comunas y sectores de la ciudad.

En esta línea, el Consejo Nacional de Desarrollo Urbano (CNDU) planteaba el año 2014 que "la mayoría de las grandes ciudades presentan altos grados de desigualdad urbana y segregación social (...) falta de acceso a servicios públicos y escasa conectividad" (p. 23).

En términos empíricos, estas diferencias se ven comprobadas en el Índice de Calidad de Vida Urbana de Comunas y Ciudades de Chile (ICVu), que realiza anualmente el Instituto de Estudios Urbanos y Territoriales (IEUT) de la PUC, el cual evalúa "las condiciones de vida de la población generadas a partir de las actuaciones y dinámicas de transformación del espacio urbano inducidas por actores públicos, privados y la sociedad civil, medidas y comparadas con información estadística provistas por fuentes oficiales públicas y privadas lo más actualizadas posibles” (IEut/Cámara Chilena de la Construcción [CChc], 2017, Presentación). En el estudio para el ańo 2017 se analizan dimensiones respecto a condiciones laborales, 
conectividad, condiciones socioculturales, medioambiente, y calidad de viviendas y entorno, entre otras. Los resultados dan cuenta, primero que todo, que de las diez grandes metrópolis analizadas, Santiago se encuentra en el octavo lugar respecto a la calidad de vida urbana de sus habitantes, lo que demostraría que existe un gran número de individuos cuyas condiciones de calidad de vida no son óptimas.

$\mathrm{Al}$ revisar de forma más detallada el análisis respecto a Santiago, se detecta la existencia de un grupo pequeño de comunas que cuentan con altos niveles de calidad respecto a las dimensiones analizadas por el estudio, incluso muy por encima de la media nacional, lo que las sitúa entre las diez mejor evaluadas. Estos territorios serían las comunas de Las Condes, Vitacura, Providencia, Lo Barnechea y Nuñoa. También dentro del rango superior de la media nacional estarían las comunas de La Reina, Santiago y Macul.

Figura I | Comunas de la Región Metropolitana entre las diez comunas del país mejor evaluadas por el ICVU 2017

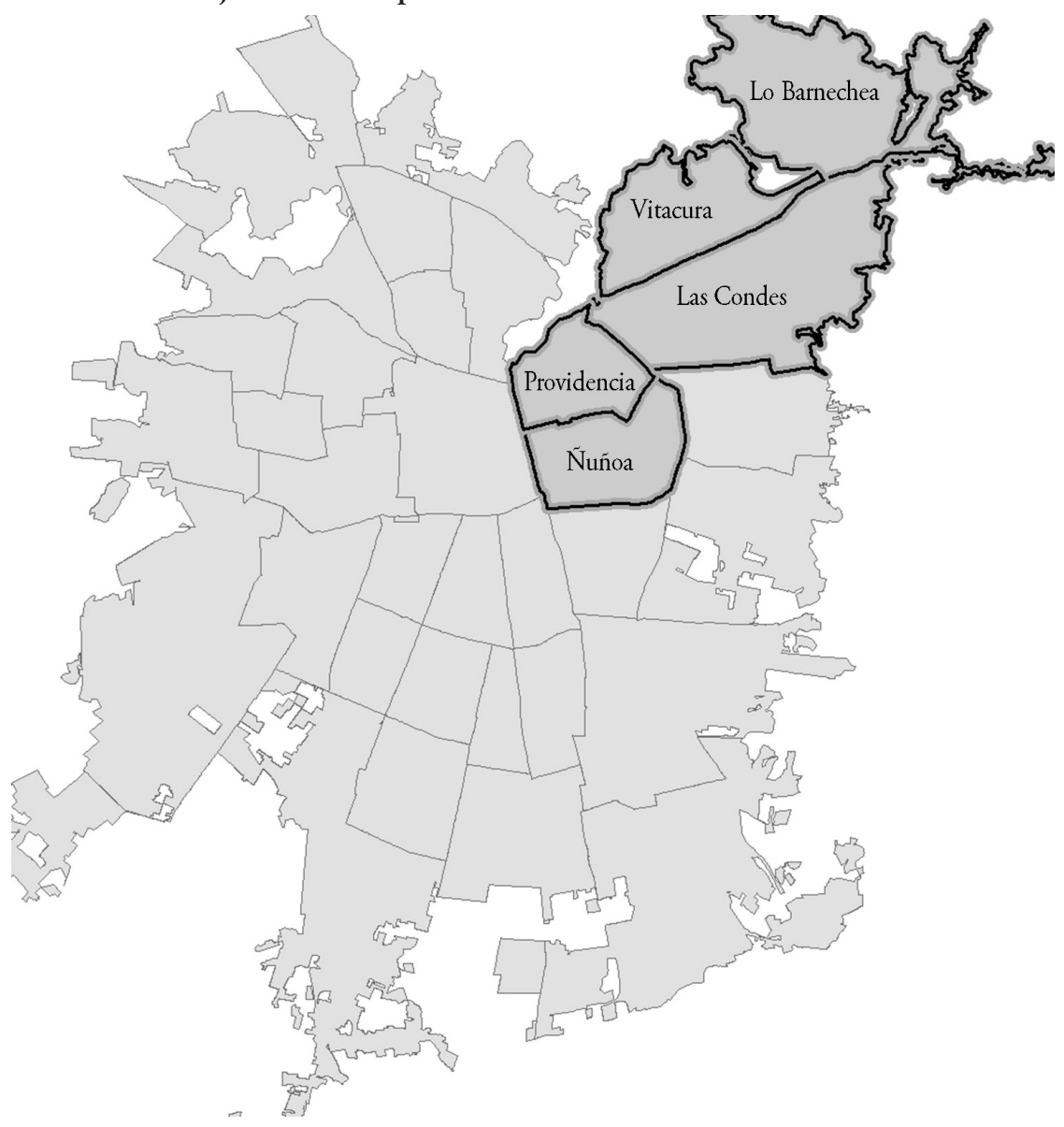

FUENTE ELABORACIÓN PROPIA A PARTIR DE DATOS PROPORCIONADOS POR ICVU (20I7) 
Por otro lado, existe un grupo de comunas que se encontraría en la vereda opuesta respecto a la calidad de vida de sus habitantes según los indicadores del estudio, en consideración a que estarían muy por debajo de la media nacional (siendo incluso las diez peores comunas a nivel nacional). Dentro de este grupo crítico se cuentan las comunas de Conchalí, Quinta Normal, Lo Prado, Independencia, San Bernardo, El Bosque, Cerro Navia, Pedro Aguirre Cerda, La Pintana y Lo Espejo.

FIgURA 2 | Comunas de la Región Metropolitana entre las diez comunas del país peor evaluadas por el ICVU 2017

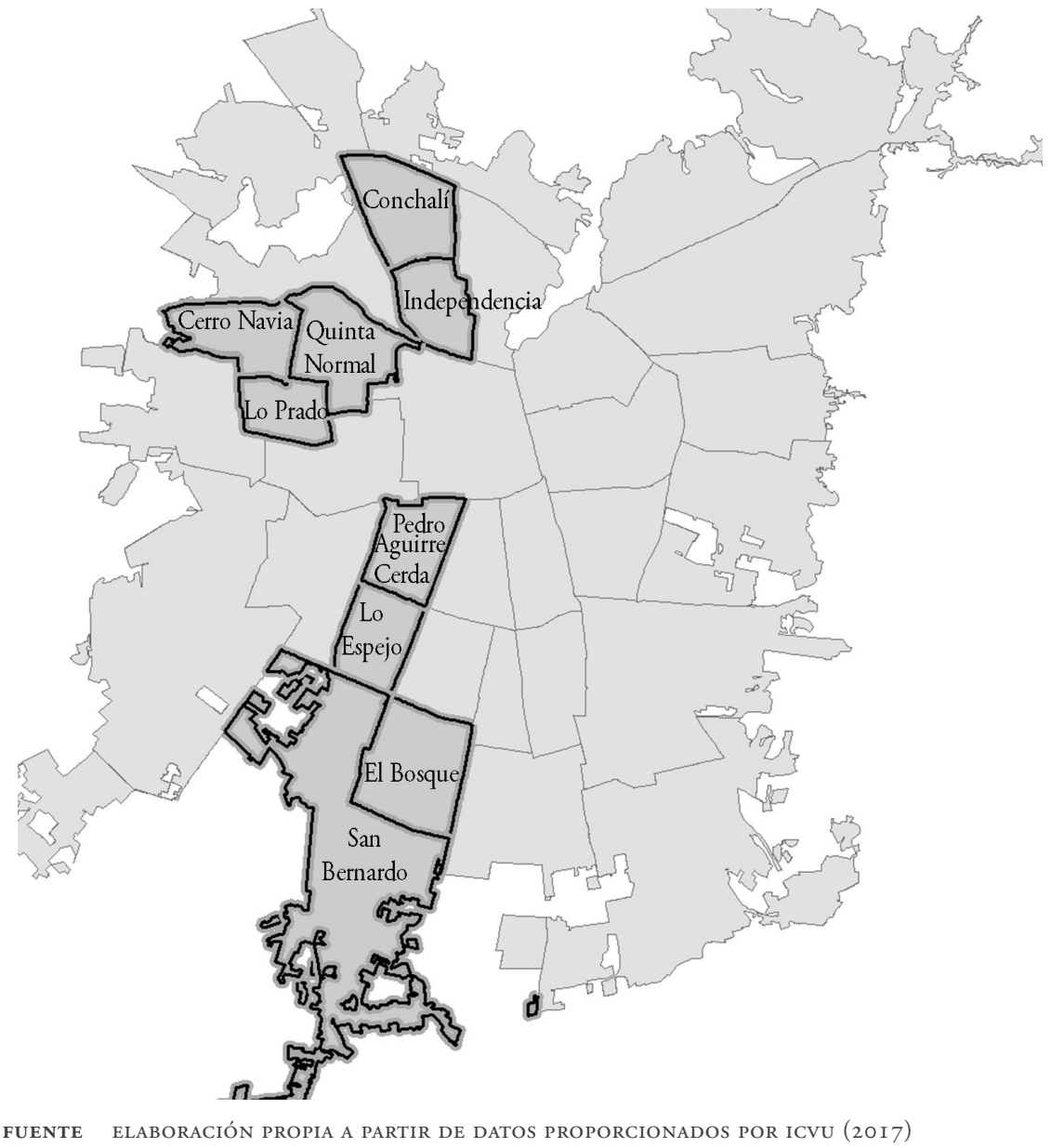

Por último, existe una capa de comunas con índices dentro del rango medio a nivel nacional. Entre ellas, Maipú, San Miguel, La Florida y Peñalolén.

En términos generales, si bien el estudio es un índice, el cual no determina cuáles son los niveles óptimos que debiese contar un territorio en específico o qué otras dimensiones debiesen entrar en consideración, sí permite sacar conclusiones 
interesantes para el punto que se quiere demostrar en este apartado. En síntesis, existe un grupo de comunas que acoge al $20 \%$ de la población del área metropolitana de Santiago, que cuenta con condiciones de vida privilegiadas a nivel nacional en función de su espacio urbano. Por otro lado, otro grupo pequeño de comunas, que comprende un $30 \%$ de la población de la ciudad, se encontraría dentro de la media nacional en cuanto a las condiciones de vida anteriormente descritas. No obstante, lo que llama poderosamente la atención es que en prácticamente un $50 \%$ de la población de Santiago las condiciones de vida urbana son inferiores a la media nacional; y, además, existe un grupo crítico de diez comunas que son las más deficientes del país.

Los datos mencionados reflejan de forma clara las diferencias territoriales que se pueden apreciar en la ciudad de Santiago, materializadas en este caso en la deficiencia en los equipamientos e infraestructura necesarios o fundamentales para que una comunidad se pueda desenvolver de forma óptima en su entorno más inmediato, como es la comuna en la cual habitan. En el caso estudiado, las diferencias materiales entre comunas son efecto de la segregación territorial socioeconómica, y obedecen principalmente a dos razones: la escasa planificación urbana de la ciudad, y la deficiencia de las políticas de vivienda aplicadas durante los años ochenta y noventa.

Autores como Rodríguez y Rodríguez (2012) acusan que uno de los grandes legados de la dictadura en materia urbana fue haber dejado la planificación urbana -entendida como la organización y planificación del crecimiento y organización de la ciudad-a merced de los intereses del mercado. Pero ello no comenzó con la dictadura. Ya para 1970, en plena consolidación del Estado neoliberal, se liberalizaba el mercado del suelo, "se simplifican las reglas para incorporar terrenos a usos urbanos y se flexibilizaron las normas que regulaban el uso de la tierra (...) para que el crecimiento urbano marchase más de acuerdo con las tendencias del mercado" (Rojas, 1999, p. 3). En esta línea, la ciudad dejaba de construirse de forma planificada y pasaba a ser regulada mediante las reglas de la oferta y la demanda. Y el Estado se restaba de evaluar dónde y cómo se debía incentivar que ciertos grupos pudieran acceder a vivir en ciertos sectores, y abandonaba la tarea de detectar qué territorios requieren procesos de regeneración urbana, o incluso creación de equipamiento, previo a que sean sectores habitables.

El resultado de las tendencias señaladas ha sido, por una parte, que al existir comunas donde se concentran grupos con considerables ingresos y capacidad de consumo, la inversión que se lleva a cabo en ellas desde el mundo privado y el mercado es alta y de calidad, proveyendo a estos territorios de un equipamiento y entorno óptimos. Y por otra parte, que los sectores donde existe una gran concentración de habitantes que no cuentan con altos ingresos y capacidad de consumo no se ven beneficiados por los equipamientos y servicios (o, por lo menos, en cuanto a su calidad) con los cuales se ven beneficiadas las clases altas.

Algunos autores se refieren a la existencia de la "precariópolis", que

sería aquel espacio monofuncional, fragmentado y segregado (...) mal conectados a la trama urbana y con infraestructura y servicios de urbanización elementales. Las viviendas son pequeñas, de baja calidad constructiva, no se observa mixtura social ni comercio y servicios de calidad (Álvarez, 2013, p. 270, citando a Hidalgo \& Orellana, 2011). 
Siguiendo con tal enfoque sobre la "precariópolis", Álvarez plantea la existencia de la "privatópolis", "que sería la ciudad suburbana y periurbana con una oferta residencial y de servicios opuesta a la anterior".

La segunda razón respecto a la diferenciación material que se evidencia en la ciudad de Santiago dice relación con la lógica de la política habitacional impuesto en la dictadura, dado que, según Rodríguez y Rodríguez (2012) se aplicó una política habitacional y de ciudad que reacomodó la organización territorial de los distintos grupos económicos. La construcción de viviendas sociales fue destinada a ser realizada por privados, restando al Estado de esta responsabilidad. Como consecuencia, al quedar la construcción de viviendas sociales en manos de privados, que tienen por objetivo conseguir el máximo de utilidades posibles y evitar mayores restricciones, los nuevos barrios construidos durante las dos décadas del régimen militar fueron llevados a la periferia de las ciudades, donde el valor del suelo tenía bajo costo. A ello se sumó la escasa calidad de las viviendas, producto del objetivo de invertir lo menos posible en construcción, y así incrementar las ganancias.

Durante el periodo señalado se construyeron innumerables conjuntos que contaban con metros cuadrados mínimos y densidades poblacionales inmensas, sin buen acceso a transporte, salud, educación y trabajo. Según Correa (2015), ello llevó a la consolidación de patrones de segregación territorial, dado que se acumulaba a la población más pobre del país en áreas determinadas de la periferia de la ciudad, con los consiguientes problemas en distintos ámbitos sociales. Un aspecto necesario de destacar es que los destinatarios de dichos conjuntos habitacionales fueron familias que en su mayoría vivían en asentamientos irregulares, pero no necesariamente en las periferias de la ciudad. Ana Sugranyes (2005) complementa este enfoque señalando:

A pesar de la obtención de la "casa propia anhelada" para los erradicados aparecieron problemas como la pérdida de empleo; el incremento de los costos, especialmente de traslado hacia y desde los lugares de trabajo y otros; dificultades de acceso a educación, salud y subsidios de la red social... (p. 33).

En conclusión, las bases sentadas por la dictadura respecto a restringir y disminuir a su mínima expresión la participación del Estado en la planificación urbana y la política habitacional, dejando ambas materias en manos del mercado y los privados, ha traído como consecuencia una ciudad como Santiago: una ciudad en la cual se evidencia una gran desigualdad territorial, donde es posible encontrar realidades disímiles en cuanto a equipamiento y calidad de los servicios según el territorio, condicionados por los niveles socioeconómicos de sus habitantes.

Entre los hogares que viven en los conjuntos más segregados es mayor el desempleo y menor la probabilidad de salir de la pobreza. Un dato revelador de ese estudio indica que la mayoría de las mujeres que habitan esos barrios populares altamente segregados prefiere no trabajar (53,8\% inactivas) puesto que la compensación monetaria proveniente del ingreso mínimo al cual ellas generalmente pueden optar, no suple el gasto tanto en tiempo como en dinero que les implica trasladarse a los lugares de trabajo (Sabatini \& Brain, 2008, p. 11). 


\section{Acumulación de la población vulnerable en determinados territorios}

Otra consecuencia de la segregación territorial remite a la homogeneización de grandes masas de grupos vulnerables en determinados sectores de la ciudad, lo que genera diferentes problemáticas de índole social, que se suman a aquellas producidas por la falta de equipamiento y acceso a la ciudad ya mencionadas.

Rodríguez \& Arriagada (2004) comentan al respecto:

La principal razón por la cual la segregación residencial está en el tapete es por las adversidades que se le imputan cuando su raíz es socioeconómica (...) [ya que] quienes, al tener como contexto cotidiano sólo pobreza y pares pobres, estrechan sus horizontes de posibilidades, sus contactos y sus probabilidades de exposición a ciertos códigos, mensajes y conductas funcionales a una movilidad social ascendente (p. 6).

El vivir en territorios vulnerables y homogéneos socioeconómicamente conlleva un fenómeno de reproducción de la pobreza, donde las posibilidades de superar una situación de vulnerabilidad se ven reducidas. Ello ocurre porque en la relación de una comunidad con su territorio se reproducen prácticas y formas determinadas de actuar en función de las problemáticas sociales dadas por el entorno. Ejemplo de ello son la deserción escolar y el trabajo delictual. En esta línea, Pierre Bourdieu, en su artículo "Efectos de lugar" (1993), plantea que habitar y poseer un espacio requiere que los actores sociales conozcan reglas y códigos particulares del lugar en cuestión; es decir, que es necesario contar con un capital cultural y un habitus específico de los distintos espacios, ya que de lo contrario se corre el riesgo de no ser aceptado por el grupo con el que se debe coexistir. Y ocurre también que si el contexto territorial se encuentra asociado a prácticas sociales permeadas por la falta de capital (laboral, cultural, social), lo más probable es que el individuo que se enfrenta a ese contexto se encuentre también permeado por esas formas de vida, lo que condicionará su actuar en la sociedad.

Otra problemática asociada a la homogenización del territorio según la condición socioeconómica, es que se construye por parte de la sociedad el etiquetado de los diferentes sectores según su condición. A su vez, este etiquetado genera mecanismos de estigmatización hacia los sectores vulnerables, los cuales operan como una barrea más para terminar con su situación de vulnerabilidad.

Pierre Bourdieu, en el libro La miseria del mundo (1993), analiza el hecho de que determinados lugares se encuentren asociados a ciertas subjetividades colectivas y se caractericen por estar ligados a valoraciones negativas o positivas, según el grupo social que dicho espacio representa. A la vez, muestra cómo diferentes estructuras espaciales se vinculan a conceptos de ascensión o declinación, materializados en percepciones como las de "subir" al barrio alto o "bajar" a los suburbios, poblaciones (para el caso chileno) o guetos.

Lo anterior se grafica con un ejercicio simple: cualquier habitante de una ciudad puede experimentar la existencia de zonas o lugares de cuyos habitantes desconfía o donde se siente extrańo o fuera de lugar, y ello por tratarse de un territorio propio de otra clase socioeconómica. Un estudio incluido en el libro Desiguales (PNUD, 2017) muestra que entre las personas que han recibido maltratos en diferentes situaciones 
públicas, un $28 \%$ considera que ello se debe al lugar en que viven, siendo la segunda razón más mencionada luego de la clase social (p. 209). Otro estudio del año 2017, realizado por el Centro de Estudios de Conflicto y Cohesión Social a nivel nacional, muestra que un 34\% de las familias de ingresos bajos y un 33\% de las familias de ingresos medios bajos cree que las personas externas a su barrio evalúan estos lugares de forma negativa o muy negativa. Ello justifica plenamente los planteamientos de Bourdieu.

De este modo, es posible concluir que la homogeneización de la pobreza -entre las distintas problemáticas que produce la segregación territorial- puede llevar, por una parte, a la formación de prácticas sociales y culturales en función de la vulnerabilidad; y por otra, a la generación de procesos de etiquetamiento y estigmatización territorial que condicionan las posibilidades de desarrollo de los individuos de determinados entornos.

\section{Beneficios de la integración territorial socioeconómica}

Diferentes expertos (Aravena, De Gregorio \& Poduje, 2013; CNDu, 2014; PNUD, 2017; Rasse, 2015; Sabatini \& Cáceres, 2004) plantean que el camino contrario a la segregación es la integración, definida ya sea como territorial, social o urbana, según el marco conceptual de los diferentes autores. Tal proceso, señalan, comporta una serie de beneficios, los cuales permiten lograr ciudades con mejores oportunidades para todos sus habitantes y con niveles de cohesión social mayores que los de ciudades segregadas.

Según los expertos, la integración territorial o urbana de una ciudad se llevaría a cabo mediante dos caminos: uno consistiría en dotar de equipamiento a sectores de la ciudad que no cuentan con condiciones materiales adecuadas para desarrollarse dentro de la trama urbana; el otro implica potenciar la creación de territorios más heterogéneos socioeconómicamente en relación con sus habitantes.

Aravena, De Gregorio \& Poduje (2013) manifiestan que para reducir la desigualdad de nuestros territorios, es necesario invertir mediante el "apoyo a los lugares y hacerlo mediante una inversión pública bien localizada, bien diseñada y mantenida en el tiempo" (p. 23). De este modo, plantean que el Estado debe revertir el tipo de desarrollo urbano que consolidó en décadas pasadas por la mera intervención del mercado, generando "inversión en sectores que muestren altos índices de segregación" (p. 23).

Por su parte, el CNDU, en la misma línea, considera que se deben definir estándares mínimos de calidad y cobertura para los bienes públicos urbanos. Una vez definidos, se deben priorizar territorios y localidades lejanas a estos niveles, para posteriormente llevar a cabo un proceso de desarrollo urbano gradual que busque proveer a todo territorio de dichos niveles de equipamiento y accesibilidad, con el objeto de mejorar la calidad de vida de todos los habitantes de la ciudad, sin discriminación alguna.

Así, queda de manifiesto que una de las principales vías para frenar la segregación y promover la integración territorial se da mediante la posibilidad de dotar a los territorios segregados de conectividad y equipamiento, con el objeto de superar la 
falta de planificación y desregulación urbana que llevaron a la creación de diferentes barrios de viviendas sociales.

Los diferentes expertos ya mencionados también consideran que se debe realizar un proceso de heterogeneización de los territorios. Lo anterior implicaría, básicamente, dos caminos: fomentar el desarrollo de viviendas de mayor valor en territorios donde existe mayor concentración de pobreza; e implementar el emplazamiento de grupos de menores recursos en sectores con buena infraestructura, conectividad y equipamiento.

Este tipo de integración y mixtura social sería provechoso por varios motivos. En el caso de incentivarse el esparcimiento de las clases más acomodadas a territorios habitados en su mayoría por grupos vulnerables, ello traerá, según diversos estudios, "el florecimiento de oportunidades de empleo y consumo para los habitantes más pobres, así como la mejora de la infraestructura" (Rasse, 2015, p. 132). Ello ocurriría, incluso, en caso de que los nuevos proyectos inmobiliarios fueran de carácter cerrado y no indujeran mayor interacción entre los diferentes grupos socioeconómicos. Lo anterior debido a que la llegada de nuevos grupos socioeconómicos de mejor situación a un determinado espacio viene asociada a incentivos para que el sector privado invierta en ese lugar, mejorando las condiciones urbanas y opciones laborales para los habitantes originales. Este proceso permite, además, que se produzca un nuevo proceso de etiquetamiento de los territorios.

Por otro lado, el hecho de que grupos vulnerables cuenten con la posibilidad de vivir en sectores mejor acomodados "trae beneficios no solo en términos de la relación con los vecinos de altos ingresos, sino también mayores oportunidades por la mejor calidad de los servicios públicos" (Rasse, 2015, p. 132). Es decir, que abre a familias con recursos escasos la posibilidad de aprovechar los beneficios de espacios ya existentes, con equipamientos y servicios de calidad, sin que sea necesaria una inversión previa del mercado o del Estado. Además, si bien Rasse (2015) plantea que no existen estudios contundentes respecto a que, con la cercanía territorial, las relaciones entre grupos se profundizan e intensifican con miras a construir ciertas redes o capital social, sí menciona la existencia de un factor de valoración positiva respecto al entorno y comuna por parte de los grupos de menores recursos, que rompe con la lógica de la reproducción de prácticas de exclusión y vulnerabilidad trabajadas por Bourdieu.

De este modo, indistintamente, investigadores, organismos públicos, políticos y organizaciones de la sociedad civil reconocen las problemáticas de la segregación territorial y los beneficios de la integración territorial. Incluso, existe por parte del Estado una política de desarrollo urbano que desde 2014 plantea la necesidad de profundizar estos lineamientos. En tales circunstancias, la pregunta que viene al caso es: ¿qué mecanismos están operando hoy en día en Chile, y en particular en la ciudad de Santiago, para promover la integración territorial de los diferentes grupos socioeconómicos?

En función de que esta pregunta es amplia y se puede responder a partir de todas las variables que se han mencionado para lograr una integración territorial, hace sentido intentar dar respuesta a por lo menos uno de los puntos considerados. De este modo, la pregunta que se trata de responder en las siguientes líneas es respecto a qué mecanismos existen y operan en la ciudad de Santiago que permiten que familias de estratos socioeconómicos bajos vivan en sectores de mayores ingresos. 


\section{Metodología}

La metodología utilizada para dar respuesta a la pregunta planteada se basa principalmente en un enfoque cuantitativo, en que se realiza una recolección de datos de segundo orden a partir de información pública disponible en la web, y la solicitud de datos mediante el sistema de transparencia por el cual se rige el Estado de Chile.

Los datos buscados y solicitados lo han sido en función de un proceso de operacionalización respecto al acceso a la vivienda en Chile, en donde se definen tres posibles mecanismos para lograr este objetivo: mercado privado, apoyo estatal y mecanismos informales. Los datos recolectados buscan ser una fotografía de la realidad que existe en Chile, y en la Región Metropolitana en particular, a diciembre del año 2017.

En cuanto a la información respecto a los valores del mercado inmobiliario, se buscó conocer los precios de venta y arriendo de viviendas por comuna, utilizando datos proporcionados por el Informe Trimestral Mercado InMobiliario Gran Santiago, de GfK Adimark, del mes de julio de 2017, y el estudio de Trivelli y Cía. (2015), Estimación y análisis de precios de arriendo residencial para las capitales regionales.

Respecto a las posibilidades de acceso a la vivienda mediante apoyo estatal, se utilizan datos proporcionados por el sistema de trasparencia solicitados al Servicio de Vivienda y Urbanización (Serviu), Región Metropolitana, e información disponible en la página web del Ministerio de Vivienda y Urbanismo (Minvu). De estas fuentes se levantó información en cuanto a los montos de los subsidios habitacionales y las comunas donde se han aplicado, ya sea en formato de compra o de arriendo, recogiendo datos del ańo 2011 a mayo de 2017.

Por último, en cuanto a la información recogida respecto a mecanismos informales de acceso a la vivienda, se utiliza información proporcionada por la fundación тесно-Chile, mediante el monitor de campamentos disponible en su página web. De allí se determina el número de campamentos existentes por comuna en la Región Metropolitana a diciembre del año 2017. Con base en lo anterior se realiza un análisis descriptivo de los datos recolectados, en el cual fue posible desarrollar tablas comunales jerarquizadas, según el valor del metro cuadrado por venta y arriendo de viviendas, número de subsidios aplicados en la comuna para compra de viviendas, y número de campamentos según comuna. Además, para el caso de los valores por metro cuadrado de venta y arriendo de viviendas, fue posible medir dichos precios en relación con el gasto porcentual que significarían para los distintos quintiles socioeconómicos, según sus ingresos promedios, proporcionados por la encuesta Casen 2015.

Una de las mayores limitantes de este análisis dice relación con que, a la fecha del estudio, no fue posible encontrar información pública y certera respecto del valor de venta y arriendo del metro cuadrado según comuna, de todos los inmuebles en venta o en arriendo. Solo fue posible considerar las unidades nuevas, lo que deja fuera un rango importante de comunas vulnerables. Además, todos los valores presentados se ven afectados por la dinámica del mercado inmobiliario y por las diferentes modificaciones de la política habitacional que van surgiendo en el tiempo. 


\section{Resultados}

El acceso a la vivienda en Chile es posible de lograr mediante diferentes mecanismos. Como primera opción está el mercado inmobiliario, donde cualquier persona que cuente con los medios económicos necesarios podrá disponer de una vivienda ya sea en arriendo o propiedad, según sus necesidades o posibilidades en función de sus ingresos. Por otro lado, existe la posibilidad de lograrlo mediante el apoyo estatal, a partir de los diferentes programas y subsidios de vivienda que el Ministerio de Vivienda ofrece. Por último, existiría un tercer mecanismo que iría por fuera del sistema formal, como es el de las tomas de terrenos públicos o privados, y la instalación en ellos de asentamientos, los que en Chile se conocen como 'campamentos'.

En cuanto a la consecución de una vivienda en el mercado inmobiliario de forma privada, el Informe Trimestral Mercado Inmobiliario Gran Santiago, de GfK Adimark, del mes de julio de 2017, muestra resultados que permiten construir un panorama global respecto a las posibilidades con que cuentan los grupos más vulnerables en cuanto a la compra de una vivienda en los diferentes sectores de la ciudad, dado que revela el valor promedio, en UF, de las nuevas unidades de vivienda que se encuentran disponibles en la ciudad de Santiago, por comuna.

Primero que todo, el informe da cuenta de una total correspondencia con el Índice de Calidad de Vida Urbano, del valor $\mathrm{UF} / \mathrm{m}^{2}$ y las comunas con mejores niveles del índice. Es decir, que ahí donde existe mayor equipamiento, conectividad y oportunidades laborales (Vitacura, Las Condes, Providencia, Lo Barnechea, Núñoa y La Reina), las viviendas (casas y departamentos) tienen un mayor valor y, por ende, son espacios a los cuales, en términos de mercado, es imposible acceder no solo para el sector más vulnerable de la población, sino que para la gran mayoría de la sociedad.

En el otro extremo, se ve que las comunas peores evaluadas en el Índice de Calidad de Vida Urbana de Comunas y Ciudades de Chile (ICvu), como El Bosque, Cerro Navia, Pedro Aguirre Cerda, La Pintana y Lo Espejo, no cuentan con precios referenciales, ya que son sectores donde el mercado privado no está desarrollando unidades de viviendas nuevas. Ello es preocupante, en el sentido de que si el mercado inmobiliario privado no incentiva la construcción de nuevos espacios que inviten a nuevos grupos a vivir en estas comunas, difícilmente se desarrollará un mejoramiento de tales territorios. Es interesante realizar un análisis más profundo de esta situación en otra investigación.

Por otro lado, entre los sectores que cuentan con la tasación más baja respecto a la adquisición de una vivienda, están comunas como Cerrillos, Conchalí, Pudahuel y Puente Alto, todas las cuales se encuentran bajo el promedio nacional del ICVu, con déficits en cuanto a vivienda y entorno. 
FIgURA 3 | Comunas de la Región Metropolitana, según precio de venta

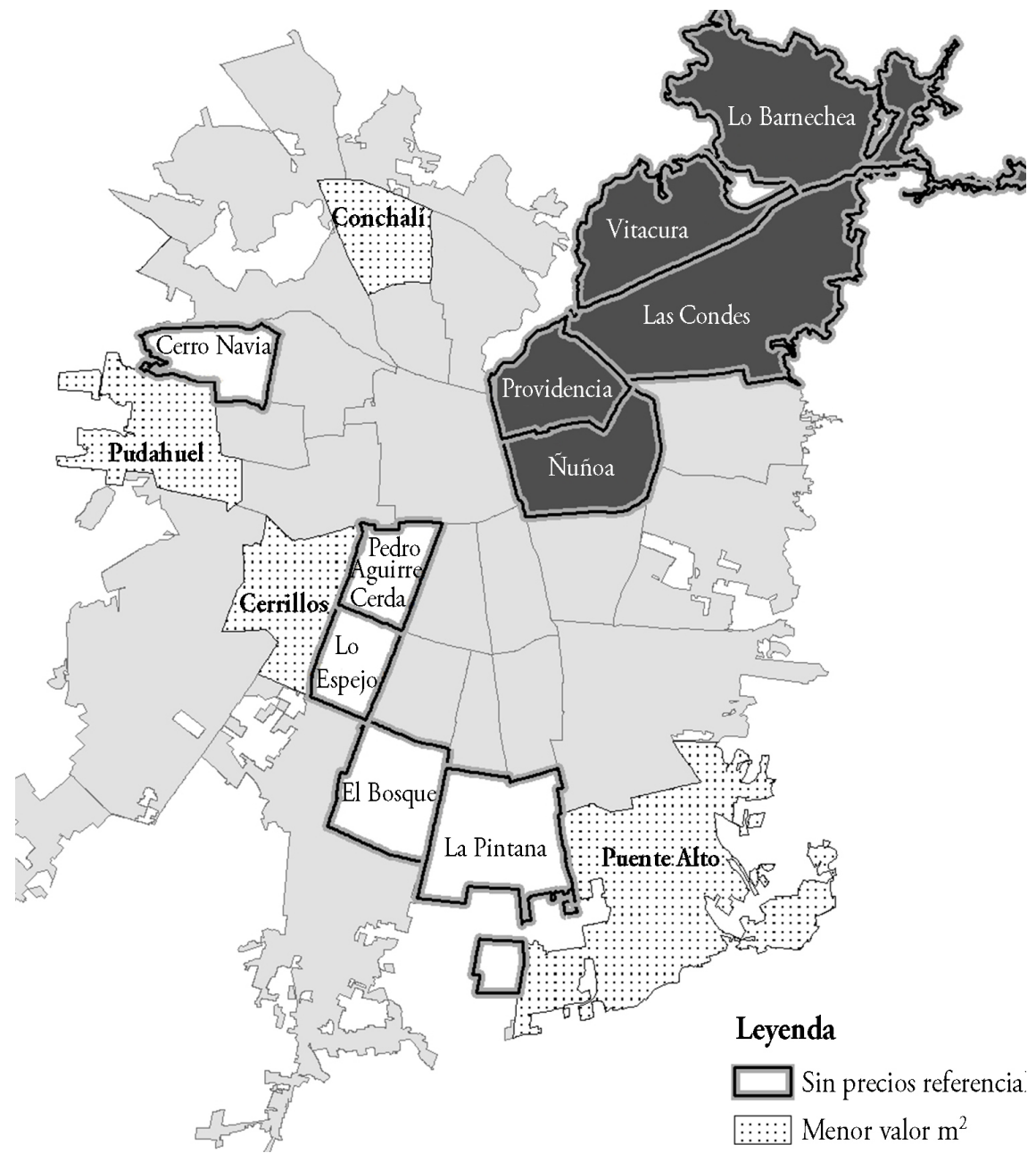

FUENTE ELABORACIÓN PROPIA A PARTIR DE DATOS DEL INFORME TRIMESTRAL MERCADO INMOBILIARIO GRAN SANTIAGO, DE GFK ADIMARK DE JULIO DE 2017

Con esta información es posible analizar las posibilidades reales que tiene una familia vulnerable de acceder a una vivienda nueva en las distintas comunas de la ciudad. En este sentido, un ejercicio simple con la comuna de Cerrillos -aquella con el precio más barato de la ciudad-permite, por ejemplo, graficar lo restrictivo del mercado de suelo actual.

La comuna de Cerrillos cuenta con un valor de metro cuadrado promedio de 34 uf en departamentos. Por ello, si una familia decide adquirir una vivienda de 42 metros cuadrados (dimensión mínima exigida a conjuntos habitacionales del Estado, no considerando subsidio de edificación en altura), debería desembolsar un 
monto que ronda los 38 millones de pesos. Así, en el caso hipotético de que dicha familia lograra concretar un ahorro del $20 \%$ (7,6 millones) y fuese posible sujeto de créditos para un banco, con una tasa de interés cercana al $4 \%$ por un período de 25 años, desembolsaría un monto mensual de 166.000 pesos, cantidad que asciende al 63\% del ingreso promedio de una familia del primer quintil de vulnerabilidad, según datos de la Encuesta Casen, y un 37\% para el caso de las familias el segundo quintil.

Por otro lado, realizando el mismo ejercicio con comunas consideradas dentro de la media nacional del ICVU, como es la comuna de San Joaquín (48,3 uf/ $\left.\mathrm{m}^{2}\right)$, el pago mensual de un dividendo ascendería a 237.000 pesos, lo cual significa el $89 \%$ de los ingresos del primer quintil, $53 \%$ de los ingresos del segundo quintil y un $37,6 \%$ de los ingresos del tercer quintil.

De este modo, es posible concluir, en términos generales, que el acceso a la vivienda en propiedad mediante los mecanismos del mercado está absolutamente restringido para los grupos más vulnerables de la ciudad, y no solo en cuanto a las comunas mejor evaluadas, sino en términos transversales a cualquier comuna de la ciudad, incluso aquellas que no cuentan con los mejores niveles de equipamiento e infraestructura, como muestra el ejercicio anterior. Tal situación constituye una de las respuestas al hecho de que en Chile aún existe casi medio millón de familias que forman parte del déficit habitacional cuantitativo.

En términos de arriendo, los datos y la conclusión son similares. Un estudio realizado por Trivelli y Cía. Ltda. del año 2016, por encargo del Ministerio de Vivienda, da cuenta de que la posibilidad de arriendo para las familias más vulnerables también es restringida dentro de la ciudad de Santiago. En este sentido, el estudio muestra que, por ejemplo, para las comunas mejor evaluadas del icvu existe solo un $0,02 \%$ de oferta en cuanto a viviendas que tengan un precio de arriendo menor a 8 UF, valor que igualmente representa prácticamente el $80 \%$ de los ingresos de las familias del primer quintil.

Por otro lado, en sectores con comunas que registran niveles de déficit en equipamiento y servicios, como La Pintana -que además concentra alta densidad de grupos vulnerables-, prácticamente no existen ofertas de alquiler, por lo cual es difícil definir un valor promedio de arriendo para estos territorios. En comunas con niveles del ICvu dentro del promedio nacional, como Peñalolén, La Florida y Macul, existe solo un 5\% de viviendas con precios de arriendo inferiores a las 8 UF.

De este modo, se da cuenta de que el mercado inmobiliario en cuanto arriendo (casas y departamentos) también es restringido para los sectores vulnerables y, una vez más, no solo en cuanto al sector preferencial de la ciudad, sino en términos generales. De hecho, datos de la Casen 2015 muestran que las familias del primer quintil de vulnerabilidad que pueden arrendar una vivienda, deben destinar un promedio de 44,9\% de sueldo a este ítem.

Queda en evidencia que, en cuanto al mercado inmobiliario, las posibilidades de integración social para las familias vulnerables son prácticamente nulas. De este modo, toma importancia el rol del Estado en lograr incluir a este grupo humano al interior de la ciudad mediante las políticas públicas de vivienda. 
Las posibilidades que tiene una familia vulnerable de encontrar apoyo del Estado en cuanto al acceso a una vivienda son variadas. Existe un subsidio para grupos vulnerables ( $40 \%$ más vulnerable), a partir del cual el Estado puede construir un conjunto habitacional (viviendas sociales), o la persona puede comprar de forma particular una vivienda nueva o usada. Por otro lado, existe un subsidio al arriendo, donde el Estado apoya a una familia con un aporte en el monto del arriendo que ella debe pagar por habitar una vivienda. Por último, existe un programa de integración, a partir del cual el Estado inserta a una familia vulnerable en proyectos inmobiliarios destinados mayoritariamente a sectores medios.

En cuanto al subsidio para grupos vulnerables destinado a la compra de forma particular de una vivienda nueva o usada, no es posible dar un diagnóstico total respecto a las posibilidades de compra que tienen los beneficiarios del subsidio. Ello debido que para el presente artículo solo se ha contado con información que considera el valor por comuna de las viviendas nuevas que se ofrecen en la ciudad de Santiago, y no de las viviendas usadas. No obstante, sí es importante considerar que en lo que respecta a viviendas nuevas, el valor máximo que permite comprar el subsidio es de 950 uf, lo que asciende a cerca de 25 millones de pesos, valor que sería insuficiente para cualquier comuna con departamentos nuevos según su valor en UF promedio, incluso considerando el comprar departamentos de $30 \mathrm{~m}^{2}$. En consideración de lo anterior, pareciera ser que, bajo la modalidad de comprar una vivienda con un subsidio habitacional, en las condiciones actuales no es posible generar un reordenamiento territorial de grupos socioeconómicos vulnerables.

Respecto a los proyectos habitacionales patrocinados por el Estado, estos cuentan con mayores oportunidades de lograr una integración territorial, dado que existen mayores oportunidades de financiamiento (cesión de terrenos públicos, financiamiento de la Subsecretaría de Desarrollo Regional (Subdere), subsidio de localización y habilitación, etcétera). Además, es posible abaratar diferentes costos según la densidad del conjunto.

A continuación se presentan las comunas donde el Estado ha construido, está construyendo o ha aprobado la construcción de proyectos habitacionales, durante el período 2011 a mayo de 2017 (figura 4).

De la figura 4 es posible rescatar diferentes conclusiones. Primero que todo, que las construcciones de viviendas patrocinadas por el Estado no se desarrollan en las comunas que lideran el ICvU; es decir, no se ejecutan en las comunas de Vitacura, Las Condes, Providencia o Nuñoa, salvo el caso marginal de Lo Barnechea y La Reina. Tampoco se reconoce la construcción de ese tipo de viviendas en comunas bien evaluadas, como Santiago y Macul.

En comunas que se encuentran dentro del promedio nacional del ICVU, es posible encontrar viviendas sociales en desarrollo o planificadas a futuro; no obstante, la presencia es más bien baja, exceptuando los casos de La Florida y San Joaquín. 
FIGURA 4 Número de viviendas sociales por comuna, aprobadas, entregadas y en ejecución

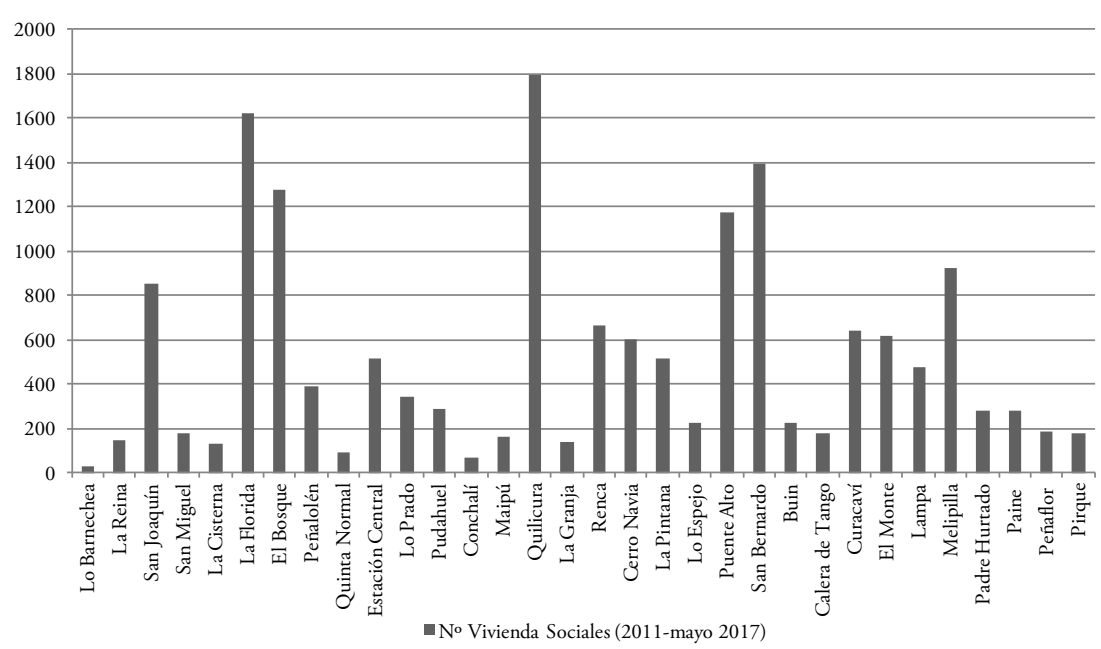

FUENTE ELABORACIÓN PROPIA, A PARTIR DE DATOS MINVU, SOLICITADOS POR TRANSPARENCIA

Por último, respecto a las comunas que se encuentran bajo el promedio nacional del ICVU, es donde más destaca la construcción reciente y futura de viviendas sociales (comunas donde, además, ya existe gran concentración histórica de viviendas sociales). Así, es posible ver cómo lideran la construcción de viviendas sociales comunas tales como Quilicura, El Bosque, Puente Alto y San Bernardo.

De este modo, se observa que los conjuntos habitacionales promovidos y financiados por el Estado se desarrollan mayoritariamente en territorios que se encuentran en una condición deficiente en cuanto a equipamientos y servicios, donde, además, existe ya una alta concentración de grupos vulnerables (en Quilicura, el $40 \%$ de la población se encuentra dentro del $40 \%$ más vulnerable, según datos Ministerio de Desarrollo Social). En este sentido, se puede concluir que el Programa para Sectores Vulnerables, en su modalidad de construcción de proyectos habitacionales, no promovería la integración territorial de grupos vulnerables a comunas con mejores niveles de calidad de vida urbana.

Otra alternativa frente a la opción de conseguir vivienda mediante el apoyo del Estado para los grupos vulnerables es el subsidio de arriendo, que cubre un monto mensual máximo de 3 uf, donde el valor máximo del total del arriendo no puede superar las 8,6 uf. El estudio realizado por Trivelli y Cía. (2015) permite identificar que si bien existen sectores donde es posible aplicar el subsidio, este es prácticamente inviable en las comunas que lideran el listado del ICvU (IEUT \& cchc, 2017).

Además, si bien los resultados del estudio muestran que sí sería posible que familias vulnerables se trasladasen desde comunas que se encuentran bajo el promedio nacional de ICvU a otras con mejor nivel, también se da cuenta de que, en su mayoría, las opciones de arriendo en estas últimas se dan en los territorios peor localizados de ellas. Como resultado, probablemente esta alternativa no signifique 
mejorar en términos objetivos las condiciones de integración territorial de las familias más vulnerables.

En consideración de lo anterior, pareciera ser que, en la actualidad, el subsidio al arriendo tampoco sería una posibilidad para lograr la integración de familias vulnerables en los espacios más desarrollados de la ciudad.

La última opción que tendría una familia vulnerable para la consecución de una vivienda mediante apoyo estatal es la ofrecida por la participación en el Programa de Integración Social. Este programa desarrolla proyectos habitacionales para familias de clase media -que reciben un aporte del Estado, pero financian la mayor parte de la vivienda mediante un crédito bancario- y para un porcentaje $(20 \%)$ de familias vulnerables, que reciben financiamiento total de la vivienda por parte del Estado. Dichos proyectos son desarrollados por Entidades Desarrolladoras, las cuales operan como inmobiliarias y, por lo general, tienen fines lucrativos.

El Programa de Integración Social y Territorial se comenzó a ejecutar durante el año 2016. A continuación se presentan las comunas en que se han llevado o están llevando cabo los proyectos correspondientes (figura 5).

\section{FIGURA 5 | Número de viviendas realizadas con subsidio DS I9, por comuna}

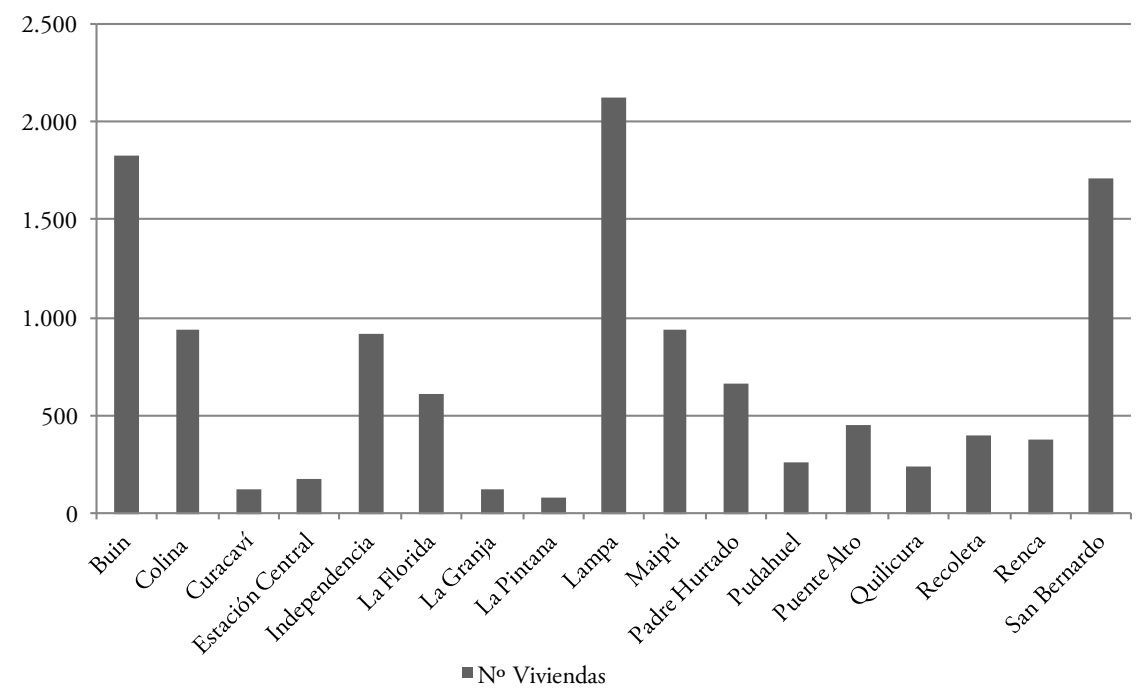

NOTA EL SUBSIDIO DSI9 ES UN PROGRAMA DE INTEGRACIÓN SOCIAL Y TERRITORIAL QUE BUSCA AMPLIAR LA OFERTA DE VIVIENDAS CON SUBSIDIO EN CIUDADES CON MAYOR DÉFICIT Y DEMANDA HABITACIONAL, A TRAVÉS DE PROYECTOS QUE INCORPOREN FAMILIAS VULNERABLES Y DE SECTORES MEDIOS, EN BARRIOS BIEN LOCALIZADOS Y CERCANOS A SERVICIOS, CON ESTÁNDARES DE CALIDAD EN DISEÑO, EQUIPAMIENTO Y ÁREA (HTTPS://IPROVIDENCIA.CL/ QUE-ES-EL-SUBSIDIO-DSI9/)

FUENTE ELABORACIÓN PROPIA, A PARTIR DE DATOS PUBLICADOS EN PÁGINA WEB MINVU

$\mathrm{Al}$ analizar las comunas donde se encuentran proyectos de este tipo, se observa que, una vez más, tampoco aparecen aquellas que lideren el ICVU. Más bien, vemos que, en su mayoría, las comunas que lideran la construcción de vivienda se encuentran 
hacia la periferia del centro de Santiago, como Buin, Lampa o San Bernardo, aunque también aparecen algunas con mejores niveles dentro del ICVU, como Maipú y La Florida. Por otro lado, es posible identificar un grupo de comunas con un indicador bajo el promedio nacional dentro del ICVU, que también cuentan con proyectos de este tipo.

En consideración de los datos presentados, es posible discernir que la política de vivienda actual para grupos vulnerables no ofrece los mecanismos que les permitan mejorar sus posibilidades de vivienda y entorno adecuados mediante el traslado a territorios con mejores equipamientos. En este sentido, no se trata solo de la falta de proyectos o posibilidades de compra y arriendo en las comunas más privilegiadas. Además, la política actual profundizaría el problema de la segregación, ya que sus programas y proyectos ofrecen mayoritariamente tan solo el acceso a una vivienda dentro de los territorios peor evaluados en cuanto a calidad de vida.

El tercer mecanismo con el cual un grupo vulnerable podría insertarse en un territorio privilegiado sería una vía irregular, como es la toma de terrenos y conformación de campamentos. Tal herramienta popular, que a lo largo de la historia de Santiago ha sido parte del proceso de conformación, expansión y consolidación de la ciudad, sigue siendo un mecanismo que ha permitido a diferentes grupos contar con mejores accesos u oportunidades en cuanto a su vivienda y entorno.

En cuanto a la creación de campamentos dentro de la Región Metropolitana, el análisis del monitor de campamentos de тесно Chile permite llegar a diferentes conclusiones (figura 6).

FIGURA 6 | Número de campamentos por comuna, Región Metropolitana de Santiago

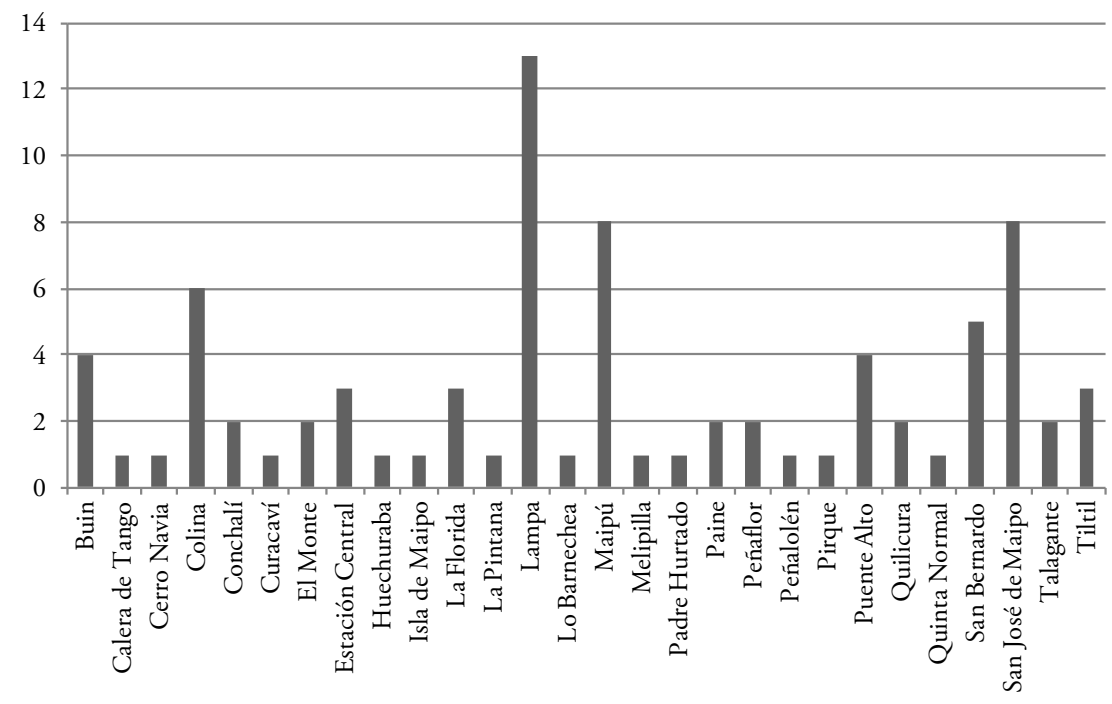

- Número de campamentos

FUENTE ELABORACIÓN PROPIA, A PARTIR DE DATOS DEL MONITOR DE CAMPAMENTOS CENTRO DE INVESTIGACIÓN SOCIAL (CIS)-TECHO. HTTP://CHILE.TECHO.ORG/CIS/MONITOR/ 
La figura 6 muestra que en la actualidad existen ochenta campamentos en la Región Metropolitana, de los cuales la gran mayoría se ubica en el sector periférico de la región, destacando comunas como Lampa, Colina, para el sector norte, y Pirque y San José de Maipo hacia el sur.

En lo que respecta a las comunas del Gran Santiago, se muestra que en aquellas que lideran el ICvU no existen campamentos, salvo en Lo Barnechea, donde existe un campamento que -según datos de TECHO- data de 1980.

En cuanto a las comunas que se encuentran dentro de la media del Índice, sí existe presencia de campamentos, pero en una cifra ínfima. La excepción es la comuna de Maipú, caso que debería analizarse con mayor detalle respecto a las razones de la cantidad de familias que viven en campamentos.

Por último, en las comunas que se encuentra por debajo del promedio nacional, sí es posible detectar mayor presencia de campamentos. Es el caso de Quilicura, San Bernardo y Puente Alto.

En este sentido, es posible concluir, en cuanto a las tomas de terreno y constitución de campamentos, que en la actualidad son más un mecanismo de acceso a un lugar donde habitar, que uno que permita a los sectores vulnerables insertarse en territorios con mejor calidad de vida urbana.

\section{Conclusiones}

Primero que todo, y a pesar de la gran cantidad de bibliografía disponible, fue necesario identificar las principales problemáticas que ha producido la segregación territorial socioeconómica. Estas se traducen, por una parte, en desigualdades en equipamiento y servicios, que afectan a los territorios más vulnerables (desigualdad permeada, además, por malas políticas públicas); y por otra, en la gran concentración de nichos de pobreza, en los cuales se reproduce la situación de vulnerabilidad, etiquetamiento y estigmatización de dichos territorios.

Por otro lado, se definen los efectos que generan los procesos de integración territorial, donde ciertos espacios se benefician con la llegada de grupos con mayores recursos, y a ciertos grupos vulnerables se les abre la oportunidad de trasladarse a territorios con mejores niveles de calidad de vida urbana. Respecto de este último punto, destacan aspectos tales como el acceso a mejores territorios en términos de equipamientos y oportunidades - produciéndose una democratización del territorio- $y$, a la vez, percepciones más positivas respecto del espacio habitado por parte de las familias vulnerables.

A partir de las opciones señaladas, se llega a la conclusión de que ninguno de los caminos que se abren está siendo una real alternativa de mejoramiento territorial y comunal para las familias más vulnerables del país. Incluso, es posible observar que las comunas con menores niveles de calidad de vida urbana son, hoy por hoy, las que siguen recibiendo al mayor número de familias vulnerables.

Estos resultados parecieran ser más bien preocupantes, dado que se observa cómo el territorio de la ciudad y la ubicación de sus ciudadanos se encuentran totalmente a merced del capital económico y no definidos a partir de un fin más social o mixto. Se repiten, así, errores del pasado que hoy en día generan un mayor gasto para el 
Estado, por la falta de oportunidades para las familias provenientes de territorios con escasa calidad de vida urbana.

Considerando lo anterior tenemos que, si bien es necesario fomentar que las comunas críticas mejoren sus niveles de transporte, vivienda, áreas verdes, equipamiento y otros, en paralelo se debe trabajar por que los territorios que ya cuentan con dichos recursos (y con municipios que también cuentan con ellos), se abran a recibir familias de menores ingresos.

Por otro lado, hace sentido cuestionarse respecto de por qué si organismos como el CNDU, o expertos en urbanismo y ciudad como Aravena, De Gregorio y Poduje (2013), plantean diferentes propuestas de política pública concretas para llevar a cabo estos planteamientos, el Estado no haya sido capaz de recogerlos y llevarlos a cabo.

Por último, es importante destacar que el presente estudio buscó ser un aporte en la temática de la integración territorial, esperando ser de utilidad para futuras investigaciones en las cuales se analicen las posibilidades de acceso a la vivienda según sector territorial, en otras regiones y ciudades del país.

Además, se espera que el artículo sea una contribución en cuanto al análisis de la política habitacional, dado que los resultados dan cuenta de que hoy el Estado chileno no está aplicando herramientas de acceso a la vivienda que permitan a las familias más vulnerables del país dejar los territorios que presentan gran concentración de pobreza.

\section{Referencias bibliográficas}

Álvarez, A. (2013). (Des) Igualdad socio espacial y justicia espacial: nociones clave para una lectura crítica de la ciudad. Polis, 12(36), 265-287. http://dx.doi.org/10.4067/S071865682013000300012

Aravena, A., De Gregorio J. \& Poduje, I. (2013). Vía rápida para reducir la desigualdad. Propuestas para revertir la segregación urbana en nuestras ciudades. Informe de Políticas Públicas 02, 1-38. https://www.espaciopublico.cl/wp-content/uploads/2016/05/14. pdf

Bourdieu, P. (1999). Efectos de lugar. En P. Bourdieu, La miseria del mundo (pp. 119-122) Buenos Aires, Argentina: Fondo de Cultura Económica.

Brain, I., Mora, P., Rasse, A. \& Sabatini, F. (2012). ¿Es posible la integración residencial en las ciudades chilenas? Disposición de los grupos medios y altos a la integración con grupos de extracción popular. EURE, 38(115), 159-194. http://dx.doi.org/10.4067/ S0250-71612012000300008

Brain, I., Prieto, J. \& Sabatini, F. (2010). Vivir en campamentos: ¿Camino hacia la vivienda formal o estrategia de localización para enfrentar la vulnerabilidad? EURE, 36(109), 111-141. http://dx.doi.org/10.4067/S0250-71612010000300005

Centro de Estudios de Conflicto y Cohesión Social (Coes) (2017). Estudio Longitudinal Social de Chile (ELSOC). Resultados Ola 1. Módulo 3: Territorio. Ciudad, conflicto y cohesión social: sobre la desigualdad socio-espacial en el Chile actual. En M. L. Méndez, G. Otero, E. López Morales \& V. Castillo. Santiago: coes. https://www.coes.cl/wp-content/ uploads/2017/08/Presentacion-Territorio-ELSOC-Ola-1-2016.pdf 
Consejo Nacional de Desarrollo Urbano (CNDu) (2014). Política Nacional de Desarrollo Urbano. Santiago, Chile: CNDU. http://cndu.gob.cl/wp-content/uploads/2014/10/L4-PoliticaNacional-Urbana.pdf

Correa, J. (2015). Crecimiento desigual: viviendas sociales en la periferia. EnfaCIS. Santiago, Centro de Investigación Social (cIs) тесно-Chile. http://www.techo.org/paises/chile/ wp-content/uploads/2016/08/\%C3\%89nfaCIS-7-Crecimiento-desigual.-Viviendassociales-en-la-periferia.pdf.

Gfk Adimark. (2017). Informe Trimestral Mercado Inmobiliario Gran Santiago. Segundo trimestre. Santiago, Chile.

Hidalgo, R. \& Arenas, F. (2011). Negocios inmobiliarios y la transformación metropolitana de Santiago de Chile: desde la renovación del espacio central hasta la periferia expandida, Revista Geográfica de América Central, (2), 1-16. http://www.redalyc.org/articulo. oa?id $=451744820395$

Hidalgo, R. \& Orellana, A. (2011). Negocios inmobiliarios y la transformación metropolitana de Santiago de Chile: desde la renovación del espacio central hasta la periferia expandida. Revista Geográfica de América Central, 2(47E), 1-16. http://www.revistas. una.ac.cr/index.php/geografica/article/view/2207

Instituto de Estudios Urbanos y Territoriales (IEUT), Pontificia Universidad Católica de Chile (puc) \& Cámara Chile de la Construcción (cchc). (2017). Índice de Calidad de Vida Urbana. Comunas y Ciudades de Chile. Santiago, Chile: IEut / cchc. http://www. asatch.cl/wp-content/uploads/2017/05/Presentacio\%CC\%81n_ICVU_2017_bj.pdf

Ministerio de Desarrollo Social, Chile (2015). Encuesta de Caracterización Socioeconómica Nacional (Casen). http://observatorio.ministeriodesarrollosocial.gob.cl/casen/casen_2015.php

Ministerio de Desarrollo Social, Chile (2017). Atlas de Acción Social. Áreas prioritarias para 75 comunas de Chile. Santiago, Chile: Ministerio de Desarrollo Social.

Orellana, A. (2017). Índice de calidad de vida urbana Comunas y Ciudades de Chile. Santiago, Chile: Instituto de Estudios Urbanos y Territoriales (IEUT), Pontificia Universidad Católica de Chile.

Programa de las Naciones Unidas para el Desarrollo (pNud) (2017). Desiguales. Orígenes, cambios y desafios de la brecha social en Chile. Santiago, Chile: PNuD http://www. cl.undp.org/content/chile/es/home/library/poverty/desiguales--origenes--cambios-ydesafios-de-la-brecha-social-en-.html

Rasse, A. (2015). Juntos pero no revueltos. Procesos de integración social en fronteras residenciales entre hogares de distinto nivel socioeconómico. EURE, 41(122), 125143. https://doi.org/10.4067/s0250-71612015000100006

Rodríguez, A. \& Rodríguez P. (2012). Políticas neoliberales en Santiago de Chile: políticas contra la ciudad. En M. Belil, J. Borja \& M. Corti (coords.), Ciudades, una ecuación imposible (pp. 131-153). Buenos Aires, Argentina: Café de las Ciudades.

Rodríguez, A. \& Sugranyes, A. (eds.). (2005). Los con techo. Un desafio para la política de vivienda social. Santiago, Chile: Ediciones SUR. http://www.sitiosur.cl/r.php?id=81

Rodríguez, A. \& Winchester, L. (2001). Santiago de Chile: Metropolización, globalización, desigualdad. EURE, 27(80), 121-139. https://doi.org/10.4067/s0250-71612001008000 006

Rodríguez, J. \& Arriagada, C. (2004). Segregación residencial en la ciudad latinoamericana. EURE, 29(89), 5-24. http://dx.doi.org/10.4067/S0250-71612004008900001 
Rojas, E. (1999). The long road to housing reform: lessons from the Chilean experience, Sustainable Development Department Best Practices Series. Washington D.C.: Banco Interamericano de Desarrollo (BID).

Sabatini, F. \& Brain, I. (2008). La segregación, los guetos y la integración social urbana: mitos y claves. EURE, 34(103), 5-26. https://doi.org/10.4067/s0250-71612008000300001

Sabatini, F. \& Cáceres, G. (2004). Los barrios cerrados y la ruptura del patrón tradicional de segregación en las ciudades latinoamericanas: el caso de Santiago de Chile. En G. Cáceres \& F. Sabatini (eds.), Los barrios cerrados en Santiago de Chile: entre la exclusión y la integración social (pp. 9-43). Santiago, Chile: Instituto de Geografía, Pontificia Universidad Católica de Chile.

Sabatini, F., Cáceres, G. \& Cerda, J. (2001). Segregación residencial en las principales ciudades chilenas: Tendencias de las tres últimas décadas y posibles cursos de acción. EURE, 27(82), 21-42. https://doi.org/10.4067/s0250-71612001008200002

Sugranyes, A. (2005). La política habitacional en Chile, 1980-2000: un éxito liberal para dar techo a los pobres. En A. Rodríguez \& A. Sugranyes (eds.), Los con techo. Un desafio para la politica de vivienda social (pp. 25-59). Santiago, Chile: Ediciones SUR. http:// www.sitiosur.cl/r.php?id=81

Trivelli \& Cía. (2015). Estimación y análisis de precios de arriendo residencial para las capitales regionales. Gran Santiago. Santiago, Chile: Comisión de Estudios Habitacionales y Urbanos, Ministerio de Vivienda y Urbanismo. http://www.minvu.cl/ opensite_20070411164455.aspx 